\title{
Frequency of Penile-Vaginal Intercourse is Associated with Verbal Recognition Performance in Adult Women
}

\author{
Larah Maunder ${ }^{1}$ (D) $\cdot$ Dorothée Schoemaker $^{2} \cdot$ Jens C. Pruessner $^{3}$
}

\begin{abstract}
Previous studies have identified a number of factors that contribute to improved cognitive function, and to memory function specifically, in cognitively normal individuals. One such factor, frequency of penile-vaginal intercourse (PVI), has been reported in a number of animal studies to be advantageous to memory for previously presented objects by increasing neurogenesis in the dentate gyrus of the hippocampus. However, studies investigating the potential benefits of frequent PVI on memory function in young women are to the best of our knowledge absent from the literature. The current study thus investigated whether the self-reported frequency of sexual intercourse was related to memory function in healthy female college students. To determine whether variation in PVI would be associated with memory performance, we asked 78 heterosexual women aged 18-29 years to complete a computerized memory paradigm consisting of abstract words and neutral faces. Results showed that frequency of PVI was positively associated with memory scores for abstract words, but not faces. Because memory for words depends to a large extent on the hippocampus, whereas memory for faces may rely to a greater extent on surrounding extra-hippocampal structures, our results appear to be specific for memory believed to rely on hippocampal function. This may suggest that neurogenesis in the hippocampus is higher in those women with a higher fre-
\end{abstract}

Larah Maunder

larah.maunder@mail.mcgill.ca

Department of Neuroscience, McGill Centre for Studies in Aging, McGill University, 6825 LaSalle Boulevard, Montreal, QC H4H 1R3, Canada

2 Department of Psychology, McGill Centre for Studies in Aging, McGill University, Montreal, QC, Canada

3 Departments of Psychology, Psychiatry, Neurology and Neurosurgery, McGill Centre for Studies in Aging, McGill University, Montreal, QC, Canada quency of PVI, in line with previous animal research. Taken together, these results suggest that PVI may indeed have beneficial effects on memory function in healthy young women.

Keywords Penile-vaginal intercourse $\cdot$ Memory Hippocampus · Dentate gyrus

\section{Introduction}

Results from animal and human studies have documented a variety of factors that may beneficially contribute to memory function. For example, environmental enrichment has proven to enhance longterm memory in rats (Bruel-Jungerman, Laroche, \& Rampon, 2005). Similarly, exercise and dietary flavonoids have been found to affect memory function advantageously in animals and humans (Berchtold, Castello, \& Cotman, 2010; Spencer, 2010).

Frequent sexual experience is another factor implicated in memory function that has emerged from animal studies. For instance, Leuner, Glasper, and Gould (2010) demonstrated that acute and chronic sexual experience enhanced cell proliferation in the dentate gyrus of adult male rats. Leuner et al. exposed the rats to sexually receptive females either once (acute sexual experience) or chronically (once per day for 14 consecutive days). Histological analyses of the number of new cells in the hippocampus suggested that a single copulation increased the number of new neurons in the hippocampus when compared to naïve controls or to rats exposed to unreceptive females. In addition, frequent sexual experience resulted in greater adult neurogenesis, as measured by a larger increase in the number of dendritic spines on dentate gyrus granule neurons, dendritic length, and the number of branch points of granule neurons compared to rats from the acute sexual experience condition.

Other studies have demonstrated a relationship between sexual behavior and neurogenesis in the dentate gyrus of 
females. Pheromones of dominant males stimulate neurogenesis in the olfactory bulb as well as the hippocampus of female mice (Mak et al., 2007). Similarly, female sheep exposed to a novel male increased the number of new cells in their dentate gyri as well (Glasper \& Gould, 2013; Hawken et al., 2009; Leuner et al., 2010). In addition, a study by Ormerod and Galea (2001) investigated the effect of estradiol and reproductive status on the rate of cell proliferation and cell survival in groups of sexually inactive and sexually active female meadow voles. Those females who were sexually active displayed a greater rate of cell survival than reproductively inactive females after 5 weeks.

The results of these studies support the hypothesis that the presence and frequency of sexual intercourse might indeed stimulate neurogenesis in the hippocampus of both males and females. Given the importance of the hippocampus in learning and memory (e.g., Gould, Beylin, Tanapat, Reeves, $\&$ Shors, 1999), and previous studies which have implicated neurogenesis in such facets of memory as pattern separation (the ability to form distinct memories of very similar stimuli that occurred closely in space or time), long-term retention of spatial memory, and recognition memory (Bruel-Jungerman et al., 2005; Clelland et al., 2009; Jessberger et al., 2009; Nakashiba et al., 2012; Sahay et al., 2011), it can be hypothesized that PVI may also be linked to improved memory function. Accordingly, in a follow-up study to their original experiment, Glasper and Gould (2013) investigated whether the neuronal proliferation that occurred in sexually experienced male rats also enhanced their cognitive function. Middle-aged retired breeder rats were exposed either to a sexually receptive female once daily for 28 consecutive days or for 14 consecutive days followed by 14 days of no sexual experience. The rats then completed novel object recognition testing, a task associated with the hippocampus (Broadbent, Gaskin, Squire, \& Clark, 2010). The results demonstrated that 28 days of sexual experience not only enhanced the number of newly generated neurons in the dentate gyrus of middle-aged rats, but also improved cognitive function. Specifically, Glasper and Gould found that the object recognition ability of these rats was similar to the performance of young adult rats and that naïve controls and those middle-aged rats that had undergone a 14-day withdrawal period after copulating for 2 weeks showed no evidence of object recognition.

Another study conducted on male mice investigated whether chronic sexual experience counteracts the negative effects of stress on adult hippocampal neurogenesis and object recognition memory (Kim et al., 2013). Kim et al. used three experimental groups of mice. The first group copulated daily with a receptive female for 3 weeks. The members of the second group also copulated daily, but were concurrently restrained for $3 \mathrm{~h}$ a day as well to induce chronic stress. The third group was also restrained daily, but did not engage in sexual activity of any sort. After the 3 weeks, the mice were tested on a novel object recognition task similar to the one conducted in the Glasper and Gould (2013) study. Mice subjected to chronic stress showed a significant decrease in hippocampal cell survival compared to controls, yet mice who were similarly stressed but also engaged in daily sexual activity displayed significantly higher levels of cell survival than the chronic stress group, levels that were not significantly different from control mice. Furthermore, the combined stress and sexual activity group also performed significantly better on the object recognition task at the 24-h retention interval than the chronically stressed mice. These results prompted Kim et al. to suggest that sexual experience could be helpful for buffering adult hippocampal neurogenesis and recognition memory function against the suppressive actions of chronic stress.

Taken together, the results from animal studies allow for the formulation of the hypothesis that frequent sexual intercourse may be beneficially associated with memory function in humans. If a comparable biological process to that which occurs in sexually active rodents also takes place in human adults, it is possible to hypothesize that more frequent PVI may be linked to increased neurogenesis in the hippocampus and superior hippocampusdependent memory performance.

\section{Possible Mechanisms for the Association Between PVI Frequency and Memory Function}

There are a number of possible explanations as to why engagement in PVI might instigate neurogenesis and/or improve memory function. Firstly, PVI is a form of physical activity, and numerous rodent studies have found associations between running, neurogenesis, and enhanced cognition (Marlatt, Potter, Lucassen, \& van Praag, 2012; Stranahan, Khalil, \& Gould, 2006; van Praag, Kempermann, \& Gage, 1999; van Praag, Shubert, Zhao, \& Gage, 2005). Secondly, it is also possible that the reward aspect of PVI may be the mechanism by which neurogenesis is promoted and memory performance is enhanced (Glasper \& Gould, 2013). The finding that exposure to dominant male pheromones both activates the female mouse reward system and stimulates neurogenesis in the dentate gyrus corroborates the supposition that the rewarding aspect of mating may enhance neurogenesis (Mak et al., 2007; Moncho-Bogani, Martinez-Garcia, Novejarque, \& Lanuza, 2005). Thirdly, engaging in sex is also associated with decreased stress and symptoms of depression (Burleson, Trevathan, \& Todd, 2007; Gordon, Burch, \& Platek, 2002). As increased stress is related to increased blunting of neurogenesis (Kim et al., 2013), and depression is related to impaired memory function and decreased neurogenesis (Burt, Zembar, \& Niederehe, 1995; Goshen et al., 2008), alleviation of both factors may result in higher memory performance.

Moreover, given that PVI increases endogenous levels of serotonin and oxytocin (Carter, 1992; Lorrain, Matuszewich, Friedman, \& Hull, 1997; Lorrain, Riolo, Matuszewich, \& Hull, 1999; Pfaus, 2009), two neurotransmitters that are involved in stimulating neurogenesis (Jacobs, van Praag, \& Gage, 2000; 
Leuner, Caponiti, \& Gould, 2012; Malberg, Eisch, Nestler, \& Duman, 2000), it is possible that these chemicals may mediate the supposed effect of PVI on neurogenesis and memory function through their increased release during PVI.

\section{Neurogenesis in Adult Humans}

The possibility that neurogenesis occurs in the adult human brain has been investigated since the early 1960s. It is now generally accepted that this phenomenon does occur in humans, due in part to the seminal work of Eriksson et al. (1998). Eriksson et al. obtained postmortem brain tissue from cancer patients who had been treated with bromodeoxyuridine (BrdU), a thymidine analog that is incorporated into the newly synthesized DNA of dividing cells, and which can be detected in their resulting daughter cells (del Rio \& Soriano, 1989; Kempermann, Kuhn, \& Gage, 1997; Kuhn, Dickinson-Anson, \& Gage, 1996). Immunohistochemical staining revealed that the dentate gyrus of the hippocampus of the patients contained BrdU-positive cells, indicating that proliferating cells are present in the adult human dentate gyrus. Additionally, to determine the cell fate of the labeled cells, they immunofluorescently labeled for BrdU and the neuronal markers NeuN, calbindin, or neuron-specific enolase. Eriksson et al. found neurons double-labeled with BrdU and each of the three neuronal markers, demonstrating that new neurons are generated in the dentate gyrus of adult humans, an ability that the hippocampus retains throughout life.

The seminal work of Spalding et al. (2013) corroborated this finding by measuring the level of nuclear-bomb-test-derived ${ }^{14} \mathrm{C}$ in the DNA of human hippocampal neurons in order to retrospectively birth date the cells. Capitalizing on the finding that ${ }^{14} \mathrm{C}$ levels in the human body mirror levels in the atmosphere, Spalding et al. demonstrated that the ${ }^{14} \mathrm{C}$ concentration in the genomic DNA of hippocampal neurons corresponds to the concentration in the atmosphere after the birth of the individual, evidencing the postnatal generation of hippocampal neurons in humans. Moreover, Spalding et al. found that only a subpopulation of hippocampal neurons, the neurons of the dentate gyrus, turn over and that approximately 700 neurons are added to this structure per day. It was concluded that the rate of adult human neurogenesis is comparable to middle-aged mice. Continued research suggests that besides the hippocampus, the subventricular zone of the lateral ventricles is also capable of neurogenesis in the adult human brain (Ming \& Song, 2011) and that these new neurons migrate to the striatum (Ernst et al., 2014).

There is also evidence that adult neurogenesis can be detected in living humans. One method developed by Manganas et al. (2007) employed magnetic resonance spectroscopy, an MRI modality, to identify neural progenitors in the live human brain, while Pereira et al. (2007) used magnetic resonance imaging and measurements of cerebral blood volume to identify an in vivo correlate of neurogenesis.

\section{Associations Between PVI and Beneficial Psychological Factors}

It is also commonly accepted that penile-vaginal intercourse is linked with beneficial states in humans. PVI has been associated with a wide range of positive psychological factors, such as relationship satisfaction (Costa \& Brody, 2007; Heiman et al., 2011), happiness (Blanchflower \& Oswald, 2004; Laumann et al., 2006; Wadsworth, 2014), lower perceived and physiological stress levels (Brody, 2006; Burleson et al., 2007), and mental health (Brody \& Costa, 2009; Kashdan et al., 2014). It is important to note that these associations do not imply causation; more sexual intercourse could be a cause, consequence, or an epiphenomenon of the above states. Though PVI's exact relation to these beneficial states is not yet conclusive, some evidence suggests that non-PVI sexual behaviors, such as masturbation, are not associated with aspects of better mental and physical health (Brody, 2010). Nevertheless, given other research that has found positive associations between masturbation and beneficial factors (Hurlbert \& Whittaker, 1991; Levin, 2007; Shulman \& Horne, 2003), it is possible that both PVI and masturbation might confer beneficial effects on memory function.

While many studies have investigated the relationship between PVI and beneficial psychological factors, systematic studies testing the possibility that PVI may be linked to neurogenesis and improved memory function in young adult humans are so far lacking from the literature. Therefore, the current study aimed to investigate the relationship between frequency of sexual intercourse and hippocampal-dependent memory function. We used a recognition memory test for our memory paradigm, as recognition memory is believed to involve the hippocampus (Glasper \& Gould, 2013; Kim et al., 2013). As proof of principle, we concentrated on assessing frequency of PVI through questionnaires, recognition memory for abstract words, and recognition memory for neutral faces in adult women. It was hypothesized that PVI frequency would be positively related to both recognition for words and recognition for faces, as encoding of both stimulus modalities relies on the hippocampus (Fernández et al., 1998; Haxby et al., 1996). We also assessed a number of additional factors we believed could also affect memory performance, including participants' school performance, menstrual cycle stage, use of oral contraceptives, frequency and intensity of exercise, non-PVI sexual activities, length of romantic relationship, and relationship strength.

\section{Method}

\section{Participants}

The sample consisted of 78 heterosexual females aged 18-29 ( $M$ age, $21.07 \pm 2.08$ years). For some of the assessments, part of the data acquisition was incomplete for various reasons (see 
below). As a result, analyses of memory for abstract words and faces were performed with $n=60$ (77\%), analyses of intimacy and sexuality were performed with a minimum of $n=30(38 \%)$, and analyses of exercise with a minimum of $n=27$ (35\%). To avoid interaction effects between the subjects' gender and the female study coordinator available for this project, only female subjects were tested. Participants were recruited via the university's psychology faculty extra course credit participant pool, as well as through the university's Classified Ads website, under "Employment/Services." These advertisements invited students to take part in a study investigating demographic variants of memory performance and stipulated that participation would involve completion of a memory test and short survey. Participants recruited through Classified Ads received \$10 in compensation upon completion of the study, and participants recruited through the participant pool obtained $1 \%$ extra credit for an eligible course within the department. The research institute's research ethics board approved the study, and informed consent was obtained from all individual participants included in the study.

\section{Procedure}

Participants were scheduled for one laboratory session. All testing took place between $10 \mathrm{~h} 00$ and $17 \mathrm{~h} 00$ on regular weekdays. Participants arrived at the laboratory for their scheduled appointment and were presented with information pertaining to the project contained in the informed consent form. After providing written informed consent, participants were presented with a computerized memory paradigm (a recognition paradigm), which required 20-30 min to complete. To minimize distractions for the participant and to avoid the participant being influenced by the awareness that she was being observed, the researcher left the room during the memory test procedure.

Once participants had completed the memory assessment, they were asked to complete an in-house questionnaire assessing participants' demographics, cumulative GPA, menstrual cycle phase, use of oral contraceptives, aspects of intimacy and sexual behavior, and exercise. This questionnaire took about 10 min to complete. After completion, participants were presented with a debriefing form detailing the purpose of the study and were provided with compensation, if eligible. The whole study took on average $<1 \mathrm{~h}$.

\section{Measures}

\section{Memory Assessment}

Participants performed a recognition task in which they were required to distinguish previously presented (old) faces and words from new (distractor) faces and words. A total of 90 neutral faces and 90 abstract words (i.e., words that were intangible nouns, such as "chastity") were used in this study. Black-and- white neutral faces were taken from Kennedy, Hope, and Raz (2009). Words with a concreteness rating lower than 4.0 were selected from Paivio, Yuille, and Madigan (1968). Concreteness ratings ranged from 1 (highly abstract) to 7 (highly concrete). A total of 60 faces and 60 abstract words were used for the encoding phase of the task, while the remaining 30 faces and 30 words were used as distractors during the recognition phase. The faces used as targets during the encoding phase and those used as distractors at the time of recognition were equalized for picture quality, ethnicity, familiarity, age, sex, and memorability across lists. The words used as targets during the encoding phase and those used as distractors were matched in length, frequency, imagery, and concreteness.

Participants were instructed to memorize faces and words that were presented to them on an Apple computer running SuperCard 4.7 (Solutions Etcetera, CA, USA). The encoding phase was performed in four encoding blocks separated by a cross-screen of $10 \mathrm{~s}$. In each block, participants were shown either a series of 15 neutral faces or 15 abstract words and were instructed to indicate whether they found each stimulus "pleasant," "neutral," or "unpleasant." Individual stimuli were presented in the middle of the computer screen for $4 \mathrm{~s}$ and were followed by a 500-ms interstimulus interval. Participants were then presented with 15 stimuli from the complementary stimulus group (i.e., words if they were shown faces before, and vice versa) and again gave a valence rating. This was followed by three more sets of 15 words and 15 faces, so that a total of 120 novel stimuli were shown. The order of faces and words was counterbalanced between participants.

The recognition phase took place immediately after the encoding phase. Participants were first presented with instructions about the forthcoming recognition procedure. Participants were then presented with all of the face and word stimuli used in the encoding phase as well as 30 new faces and 30 new words, which served as distractors. Therefore, a total of 180 faces and words were presented in the recognition phase. Stimuli appeared in three groupings of 30 words and 30 faces, in randomized order. Subjects were asked to indicate whether the word or face depicted on the screen was new or old.

A sensitivity score represented each participant's recognition performance on the recognition paradigm. The sensitivity score was calculated separately for abstract word recognition and face recognition using the number of hits and false alarms the participant made during the recognition phase of the memory test. The correct indication of a previously presented stimulus as old constituted a hit, while a false alarm was defined by the incorrect indication of a new stimulus (a distractor) as old. Sensitivity scores were then calculated by computing the difference between the percent of old stimuli correctly identified and the percent of distractors incorrectly accepted as old. Therefore, higher sensitivity scores represent superior recognition performance (Table 1). 


\section{Demographics}

We assessed participants' age, height, weight, and sexual orientation through use of an in-house questionnaire. Further, participants provided their cumulative grade point average (GPA), as there is substantial evidence for a positive relationship between GPA and intelligence (Richardson, Abraham, \& Bond, 2012), of which memory function is one important aspect. In addition, participants reported the first day of their last menses, if known, to allow calculation of their menstrual cycle phase. Likewise, the use of oral contraceptives was assessed by the survey question "Do you take oral contraceptives?"

\section{Intimacy and Sexuality}

The in-house survey investigated the individual's experience of intimacy with 3 items. These items inquired about the type and duration of the participant's relationship and included an assessment of the depth of affection she felt for her partner (if the individual was involved in a romantic relationship). Possible relationships included committed monogamous, semimonogamous, casual dating, single and sexually active (including sexual intercourse), single and sexually active (excluding sexual intercourse), and single and not engaging in sexual activity of any kind. Those participants who reported that they were in a committed monogamous relationship, a semi-monogamous relationship, or were casually dating a partner recorded the length of their current relationship and completed a series of questions measuring their experience of romantic love. Here, participants indicated the nature of the romantic relationship that they were currently engaged in, by completing the Measurement of Romantic Love Scale (Rubin, 1970). The scale measures the participant's affiliative and dependent need for her partner, her predisposition to help him, and the exclusiveness and absorption she feels for him. Response items for the Measurement of Romantic Love Scale are found in “Appendix." Two pools of participants were established from the women who completed this questionnaire. Participants who scored above the median on the Love scale were designated as being in a "strong love" relationship, while those who scored below the median were designated as being in a "weak love" relationship.

In addition, the in-house questionnaire contained 6 items that assessed participants' current (within the last month) sexual behaviors, including whether or not they engaged in sexual intercourse, how often they engaged in sexual intercourse, how often they partook in other partnered sexual activities excluding PVI, whether their partner intravaginally ejaculated during PVI without a condom, how often they achieved orgasm from PVI, and whether and how often they masturbated. Sexual behavior questionnaire items are found in "Appendix." Not all participants answered all of these questions as completion of all items on the questionnaire was voluntary, and because not all items were applicable to all participants (for instance, if a participant indicated that she did not engage in PVI, the question of ejaculation did not apply). Therefore, this additional information was only available in part of the study group. In 59 participants, information about the frequency of non-PVI partnered sexual activity was available. Information about ejaculation during PVI was available in 31 of these participants, use of condoms during PVI in 38, orgasm during sexual intercourse in 32, and masturbation in 30.

\section{Exercise}

Finally, we also assessed the amount of physical exercise a subset of participants engaged in per week. Participants indicated the length of time (in minutes) they spent exercising, the intensity of the exercise (high, medium, or low), and how often they exercised per week.

\section{Analyses}

The main analysis aimed to investigate the association between memory performance and the frequency of sexual intercourse and to establish whether there was any significant association between these two measures. To obtain a proxy of memory, we employed the sensitivity scores from the recognition paradigm, separately for abstract words and neutral faces. We performed a multiple linear regression with sensitivity scores as the criterion variable, and frequency of sexual intercourse, menstrual cycle phase, grade point average, use of oral contraceptives, and relationship length as predictors. Menstrual cycle phase was dummy coded into " 1 ," corresponding to the follicular phase of their menstrual cycle (Day 1-14) or "2," representing the luteal phase of their menstrual cycle (Day 15-28), where Day 1 was their first day of menstruation.

\section{Results}

\section{Words}

Association of Sexual Intercourse Frequency with Sensitivity Scores for Abstract Words

The mean sensitivity score for abstract words was .70, with a SD of \pm .16. Sensitivity scores for the sample ranged from .33 to 1.00 . Performing a multiple linear regression with sensitivity scores as the criterion variable, frequency of sexual intercourse as the predictor variable, and entering participants' GPA, phase of menstrual cycle, oral contraceptive use, and relationship length as additional predictors yielded a significant regression model with $R^{2}=.19, F(5,55)=2.65, p=.032$. As hypothesized, PVI frequency was positively associated with abstract word sensitivity scores on the memory paradigm. In the model, only PVI frequency was a significant predictor, $\beta=.49, t(55)=3.45$, 
Table 1 Summary of multiple regression analyses for variables predicting sensitivity scores for words and sensitivity scores for faces

\begin{tabular}{|c|c|c|c|c|c|c|c|c|c|c|c|c|}
\hline \multirow[t]{2}{*}{ Variable } & \multicolumn{9}{|c|}{ Sensitivity words } & \multicolumn{3}{|c|}{ Sensitivity faces } \\
\hline & $B$ & SE $B$ & $\beta$ & $B$ & SE $B$ & $\beta$ & $B$ & SE $B$ & $\beta$ & $B$ & SE $B$ & $\beta$ \\
\hline PVI frequency & 0.44 & 0.01 & $0.49 * *$ & & & & & & & 0.01 & 0.01 & 0.15 \\
\hline Oral contraceptive use & 0.07 & 0.05 & 0.21 & -0.03 & 0.05 & -0.11 & & & & 0.04 & 0.05 & 0.11 \\
\hline GPA & -0.04 & 0.05 & -0.10 & -0.001 & 0.06 & -0.003 & & & & -0.07 & 0.06 & -0.17 \\
\hline Menstrual cycle phase & 0.02 & 0.04 & 0.04 & 0.02 & 0.05 & 0.06 & & & & 0.03 & 0.05 & 0.10 \\
\hline Relationship length & -0.01 & 0.02 & -0.05 & -0.01 & 0.02 & -0.04 & & & & 0.001 & 0.02 & 0.005 \\
\hline Frequency of orgasm attainment during PVI & & & & & & & 0.04 & 0.02 & $0.52 *$ & & & \\
\hline Frequency of sexual activity other than $\mathrm{PVI}^{\mathrm{a}}$ & & & & -0.01 & 0.01 & -0.16 & & & & & & \\
\hline$R^{2}$ & 0.19 & & & 0.049 & & & 0.27 & & & 0.055 & & \\
\hline$F$ & $2.65^{*}$ & & & 0.41 & & & $4.78 *$ & & & 0.64 & & \\
\hline
\end{tabular}

$* p<.05 ; * * p=.001$

${ }^{a}$ Regression conducted for women who do not take oral contraceptives

$p=.001$, while the other factors did not reach significance. This suggests that $19 \%$ of the variability in abstract word sensitivity scores was explained by the model. Since sensitivity scores are derived from the difference between hits and false alarms, this relationship is also represented in the correlation between frequency of PVI and number of hits and false alarms. For hits, a positive correlation with frequency of sexual intercourse, $r=.34$, $p=.009, n=61$, was observed, while the number of false alarms was negatively correlated with frequency of sexual intercourse, $r=-.28, p=.035, n=61$ (Figs. 1, 2).

We next wanted to explore whether the frequency of engaging in partnered sexual activity other than intercourse would have a similar effect on the sensitivity scores for abstract words. For that purpose, we entered frequency of partnered sexual activity other than PVI, menstrual cycle phase, oral contraceptive use, GPA, and relationship length into a regression model as predictor variables, with sensitivity scores for words employed as the criterion variable. No significant effect could be observed for this analysis, $R^{2}=.049, F(5,40)=.41, p>.20,1-\beta=.16$, and frequency of partnered sexual activity other than PVI did not significantly predict sensitivity scores, $\beta=-.16, t(40)=-.96, p>.20$.

\section{Association of Orgasm Attainment During PVI with Sensitivity Scores for Abstract Words}

To investigate whether orgasm frequency might be related to memory, a regression analysis was conducted for the relationship between frequency of orgasm during PVI and memory performance. Frequency of orgasm during PVI was employed as the predictor variable, and sensitivity scores for abstract words were used as the criterion variable. The regression analysis was conducted separately for women who were not taking oral birth control, and women who were. The regression model for women who were not taking oral contraceptives was significant, $R^{2}=.27$, $F(1,13)=4.78, p=.048$, and orgasm frequency during PVI sig-

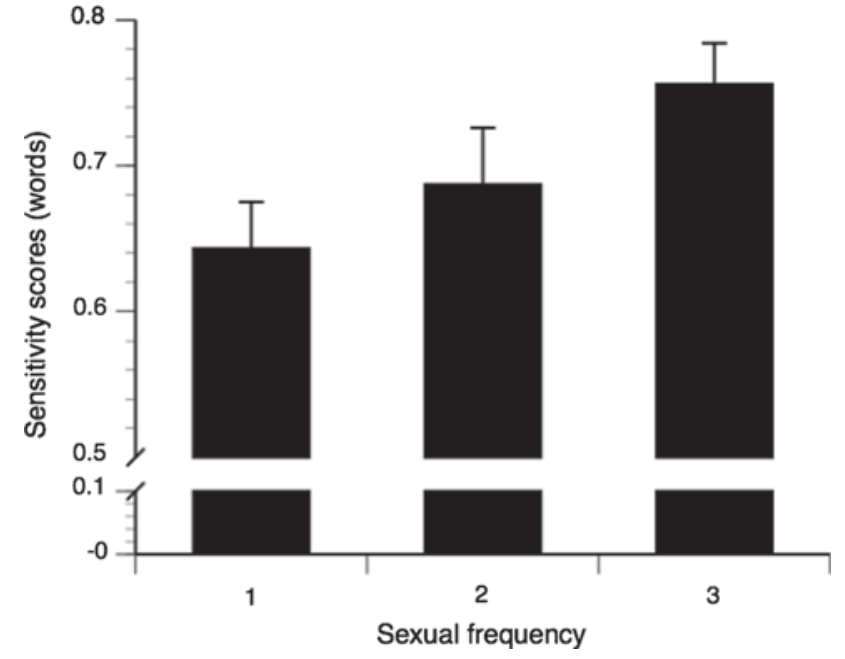

Fig. 1 PVI frequency and abstract word sensitivity scores are positively associated in heterosexual women $[F(5,55)=2.65, p=.032, n=60]$. Group 1 represents women who do not engage in PVI or do so less than once per month. Group 2 represents PVI approximately once per month up to once every 2-3 weeks. Group 3 represents PVI once per week to once per day. The $y$-axis shows sensitivity scores (hits-false alarms) for remembering abstract words. Higher values suggest better memory

nificantly predicted sensitivity scores for abstract words, $\beta=.52$, $t(13)=2.19, p=.048$. Frequency of orgasm achievement during PVI was not significantly associated with abstract word sensitivity scores for women who were taking oral contraceptives, $F(1$, 17) $<1, p>.20,1-\beta=.08$.

\section{Faces}

Association of Sexual Intercourse Frequency with Sensitivity Scores for Neutral Faces

The mean sensitivity score obtained for faces was .61 \pm .16 , with individual scores ranging from .21 to .90 . No significant 


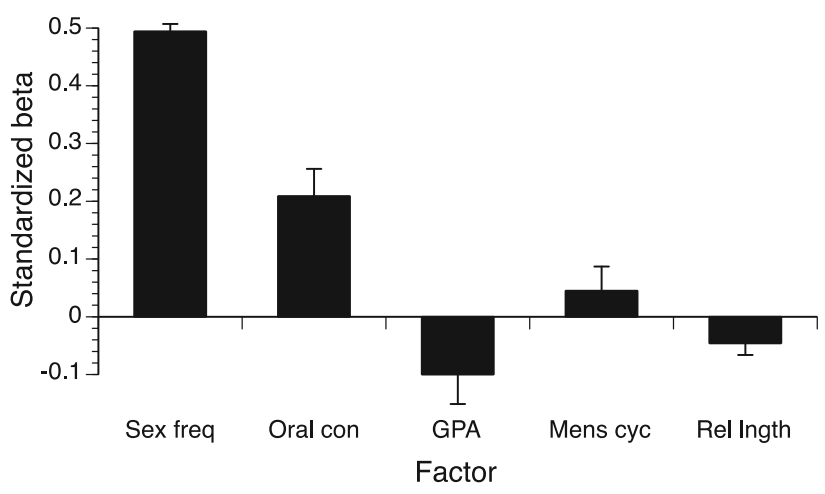

Fig. 2 Sensitivity scores for abstract words are related to PVI frequency for heterosexual women $[F(5,55)=2.65, p=.032, n=60]$. Standardized betas are shown for all predictor variables in the model. PVI frequency was the only significant predictor in the model, $\beta=.49$, $t(55)=3.45, p=.001$. Sexfreq frequency of sexual intercourse, Oral con oral contraceptive use, GPA cumulative grade point average, Mens cyc menstrual cycle phase; Rel lgnth relationship length

association was obtained between women's recognition scores for faces and PVI frequency when a multiple linear regression was conducted between these variables, again holding constant the possible confounds of grade point average, menstrual cycle phase, use of oral contraception, and relationship length, $R^{2}=$ $.055, F(5,55)=.64, p>.20,1-\beta=.24$. PVI frequency also did not significantly predict sensitivity scores, $\beta=.15, t(55)=$ $.96, p>.20$. Similarly, no significant correlation was obtained when a partial correlation was conducted between frequency of PVI and hits, and between frequency of PVI and false alarms, $r=.08, p>.20, n=61,1-\beta=.10$, and $r=-.09, p>.20$, $n=61,1-\beta=.11$, respectively.

\section{Other Factors and their Relation to Memory Performance}

No group means (strong love, weak love, no romantic relationship) were significantly different when a one-way ANOVA was conducted on relationship strength group and abstract word memory scores, $F(2,65)=1.79, p=.175,1-\beta=.36$. Further, no significant correlation was observed between GPA and scores on the recognition paradigm for abstract words, $r=$ $-.11, p>.20, n=62,1-\beta=.14$. Also, a one-way ANOVA was conducted for menstrual cycle phase and corresponding abstract word sensitivity scores. This analysis was completed only for women who were not on oral birth control. Women in their luteal phase $(n=21)$ did not differ in sensitivity scores from women in their follicular phase $(n=12), F(1,31)<1$, $p>.20,1-\beta=.06$. An ANOVA was also conducted for the use of oral contraceptives and sensitivity scores, and again, no significant difference was obtained between the two variables, $F(1,66)<1, p>.20,1-\beta=.06$.

Similarly, a linear regression analysis with frequency of exercise per week, duration of exercise, and intensity of exercise did not reveal any significant association with abstract word sensitivity scores on the memory paradigm, $F(3,24)<1, p>$ $.20,1-\beta=.13$.

Finally, frequency of masturbation per week, $r=-.07$, $p>.20, n=30,1-\beta=.07$, frequency of orgasm achievement from masturbation, $r=-.14, p>.20, n=30,1-\beta=$ .12 , and frequency of intravaginal ejaculation $r=.11, p>.20$, $n=31,1-\beta=.09$, were all not significantly associated with abstract word sensitivity scores, while controlling for GPA, phase of menstrual cycle, and oral contraception use.

\section{Discussion}

The current study found a significant association between memory scores for recognition of abstract words (measured via sensitivity scores) and frequency of PVI, while holding constant menstrual cycle phase, grade point average, use of oral contraceptives, and relationship length in a population of young female undergraduate students. No such association was found for neutral faces. Furthermore, a significant association was also found between memory scores for abstract words and frequency of orgasm achievement during PVI for those women who did not take oral birth control.

These findings support the initial hypothesis that frequency of sex would be positively associated with memory scores. While the current findings are correlational, previously conducted animal studies suggested a causal link by experimentally controlling the frequency of sexual intercourse, observing an effect on memory performance, and showing signs of increased neurogenesis in the hippocampus (Glasper \& Gould, 2013). It can be speculated whether the neuroanatomical changes that occurred in sexually active rodents similarly take place in humans as a result of having PVI as well. As hippocampal neurogenesis has now conclusively been shown to occur in the human adult dentate gyrus (Eriksson et al., 1998), and because evidence suggests that these neurons integrate themselves into the existing neural network to become functional (van Praag et al., 2002), it is possible that lifestyle factors such as PVI frequency contribute to increasing neuronal proliferation in both men and women, just as it does in rodents.

To provide causal evidence for this relationship in humans would require randomized, controlled experiments. This could possibly be combined with longitudinal studies measuring memory performance in heterosexual and LGBTQ2 adults over an extended period of time, while simultaneously monitoring frequency and type of sexual activity (such as penile-anal intercourse in gay men). Such studies may determine whether memory performance fluctuates with participants' changing levels of PVI and other sexual behaviors over time. They may also determine whether the superior memory performance demonstrated by participants who engaged in frequent PVI endures even if PVI frequency declines or ceases. This research could also include structural neuroimaging (e.g., using MRI) of the hippocampus and neighboring medial temporal lobe structures and subsequent 
volumetric analyses. Such research could more clearly demonstrate the role that intercourse and other sexual activity frequency have on the memory performance of sexually active individuals.

It was interesting to observe that frequency of sexual intercourse was only associated with memory scores for abstract words but not with those of faces. We can interpret this observation as a sign that the relationship between frequency of sexual intercourse and abstract word sensitivity is indeed associated with a hippocampus-dependent memory function. Current research suggests that successful face recognition may depend to a greater extent on extra-hippocampal structures such as the fusiform face area (FFA) in the fusiform gyrus, the occipital face area (OFA) in the inferior occipital gyrus, and anterior temporal cortical areas (e.g., Nestor, Plaut, \& Behrmann, 2011; Rossion et al., 2003; Rossion, Hanseeuw, \& Dricot, 2012). Neuroimaging studies have demonstrated that successful word retrieval, however, depends on the recruitment of the hippocampus (Heckers, Weiss, Alpert, \& Schacter, 2002). In addition, it can be argued that faces present a more complex stimulus set, requiring the encoding of more characteristics to allow accurate recollection. Thus, it may be more likely that participants had to rely on the perception of familiarity when asked to recollect face stimuli, a process that is disproportionately associated with activity in the perirhinal cortex rather than the hippocampus (Diana, Yonelinas, \& Ranganath, 2007). Therefore, it can be speculated that memory for abstract words was dependent on hippocampal activation to a greater extent than memory for faces, which relied more on surrounding structures such as the FFA, OFA, and perirhinal cortex. As sexual intercourse has been shown to contribute to neurogenesis in the dentate gyrus of the hippocampus, at least in animals (Glasper \& Gould, 2013), this dissociation may explain the absence of correlation between PVI frequency and face recognition.

While the current findings provide first evidence for a possible link between frequency of PVI and memory for abstract words, the study had a number of limitations. Most importantly, the current findings were correlational and do not allow for the establishment of a cause-effect relationship. Also, the results were based on a convenience sample, allowing for naturally occurring variations that might explain the current results without being related to the frequency of PVI. In addition, the assessment of sexual activity was done retrospectively, and via self-assessment, allowing for inaccuracies in the reporting of this information. In order to minimize potential inaccuracies in the reporting of sexual activity, future studies should obtain questionnaire responses from both persons in the romantic partnership (if the participant is in a romantic relationship) in order to test for concordance in the responses of both individuals, or have respondents record their sexual activity daily over the course of a month.

A number of additional observations with regard to the study sample and its results should be noted. Importantly, there was no significant relationship between masturbation frequency or achievement of orgasm during masturbation and abstract word recognition scores. According to Brody (2010), this result would be expected since PVI is the only sexual behavior directly relevant to gene propagation, and because evolutionary pressures strongly reward behaviors and mutations associated with an increased likelihood of gene propagation. Thus, only PVI would become associated with better physical and mental health indices, such as memory function and/or hippocampal neurogenesis, over evolutionary time. The observation, for example, that prolactin release was $400 \%$ greater after intercourse as compared to masturbation in one study provides independent support for this line of argumentation (Brody \& Krüger, 2006). On the other hand, methodological concerns regarding Brody and Krüger's findings have been voiced (Levin, 2007), making it difficult to conclude whether PVI truly was more strongly associated with a beneficial factor than masturbation in this study. In the current study, as the sample size for masturbation frequency and attainment of orgasm during masturbation (as well as the sample sizes for intravaginal ejaculation and exercise) were lower than the total sample size, a small effect of these parameters on memory function may still exist, but might have been missed due to the low power of the sample. Given that positive relationships have been found between masturbation and beneficial states, such as masturbation being associated with positive body image for European American women (Shulman \& Horne, 2003), and evidence for greater marital and sexual satisfaction among married women who masturbate to orgasm (Hurlbert \& Whittaker, 1991), it is still plausible that a positive association exists between masturbation frequency and memory performance as well. Future studies with a larger sample size should be conducted to better investigate this potential relationship.

Importantly, it is also possible that other social and nonPVI related variables might account for the significant association demonstrated in this study. However, there was no significant association found between the strength or length of romantic relationship and memory scores. Thus, those single women who self-reported a high frequency of sex demonstrated higher memory scores than those coupled women who self-reported either not having sex or having a low frequency of sex. This suggests that it is PVI, and not the presence of a relationship, that accounts for the beneficial effects found in this study. Similarly, the lack of a significant relationship found between the predictors GPA, menstrual cycle phase, and use of oral contraceptives and word recognition scores supports the conclusion that engaging in PVI is the most decisive factor in accounting for the variation in memory performance.

There are a number of mechanisms by which the demonstrated effects of sexual behavior on memory function may occur. For one, PVI is a form of physical activity, and numerous rodent studies have found associations between various forms of exercise, neurogenesis, and enhanced cognition (Marlatt et al., 2012; Stranahan et al., 2006; van Praag et al., 1999, 2005). Exercise potentially improves brain health through increasing levels of various molecules in the brain, such as brain-derived 
neurotrophic factor (BDNF), various neurotransmitters, and specific hormones (Cotman \& Berchtold, 2002). One study on elderly adults reported that the positive correlation between physical fitness and spatial memory performance was partially mediated by hippocampal size (Erickson et al., 2009). While not a direct measure of hippocampal neurogenesis, it was suggested that cell proliferation may be one factor which increases hippocampal volume measurements specifically in physically active older adults. Thus, it may be that the physical activity component of PVI promotes neurogenesis and increases memory function in adult women.

However, as PVI is generally regarded as a rewarding experience, it is possible that the reward aspect of PVI may be the mechanism by which neurogenesis is promoted and memory performance is enhanced (Glasper \& Gould, 2013). This may be especially so given that dopamine (a neurotransmitter implicated in reward) may promote neurogenesis in the hippocampus (Becker, Rudick, \& Jenkins, 2001; Takamura et al., 2014), and because sexual activity activates reward centers in the brain (Ågmo \& Berenfeld, 1990; Damsma, Pfaus, Wenkstern, Phillips, \& Fibiger, 1992). The finding that intracranial self-stimulation enhances hippocampal neurogenesis in rodents corroborates this finding (Takahashi et al., 2009). In addition, some recent evidence suggests that sexual activity might increase neurogenesis in the striatum(Bedard, Gravel, \& Parent, 2006; Dayer, Cleaver, Abouantoun, \& Cameron, 2005; Ernst et al., 2014), thereby further increasing the reward salience of sexual behavior (Báez-Mendoza \& Schultz, 2013; Takamura et al., 2014).

In support of the argument for the rewarding rather than the physical activity component of PVI, it has been found that rats subjected to forced treadmill running display physiologically maladaptive changes indicative of chronic stress, such as adrenal hypertrophy and adrenal involution (Moraska, Deak, Spencer, Roth, \& Fleshner, 2000). As neurogenesis in the hippocampus is diminished by stress (Mirescu \& Gould, 2006), it is possible that the voluntary aspect of running, considered to be rewarding in rodents (Greenwood et al., 2011; Meijer \& Robbers, 2014), must be present in order to buffer the brain against the deleterious effects of stress and to promote neurogenesis. Such a possibility lends credence to the proposition that it is specifically the rewarding aspect of PVI that promotes increases in neurogenesis and cognitive function in women (Báez-Mendoza \& Schultz, 2013; Becker et al., 2001; Mermelstein \& Becker, 1995; Pfaus, Damsma, Wenkstern, \& Fibiger, 1995).

PVI has also been associated with a number of psychological factors that may mediate the relationship found in the current study. For instance, sexual behavior is positively related to mood in women and inversely related to depressive symptoms in women who have sex without condoms (Burleson et al., 2007; Gordon et al., 2002). Given that a significant, stable association between depression and impaired memory function has been found (Burt et al., 1995), it is possible that women who self-report having more intercourse experience less depression, which results in better memory performance. Moreover, depression is associated with impaired neurogenesis (Goshen et al., 2008), leading to the possibility that women who engage in more frequent sex not only experience less depressive symptoms, but may also benefit from increased neurogenesis in their hippocampi and, consequently, improved memory function.

With regard to stress and anxiety, Kim et al. (2013) found that, in mice, sexual intercourse counteracted the detrimental effects of chronic stress on hippocampal neurogenesis. In women, sex may also be beneficial for decreasing anxiety and stress, as negative associations have been found between these factors and PVI (Brody, 2006; Burleson et al., 2007; Kashdan et al., 2014). For instance, women report experiencing lower levels of anxiety symptoms the day after engaging in sex than when they did not have sex the previous day (Kashdan et al., 2014). Thus, it is possible that women who engage in sex benefit from higher rates of neurogenesis due to the decreased levels of stress and anxiety symptomatology they experience.

It is possible that, aside from psychological factors, the time of day during which participants completed the memory task may have contributed to variance in memory scores, as cognitive performance might differ from morning to night. Future studies might benefit from counterbalancing appointment times between individuals who engage in PVI at high and low rates, such that an equal number of participants who engage in PVI at a high rate and a low rate are tested during morning appointments and during afternoon appointments.

The specific biochemical mechanism that may drive the neurogenesis posited to occur as a result of PVI remains to be elucidated. Serotonin, one possible biological mediator, is primarily involved in the central regulation of the sexual response (Rowland, 2006). Current evidence indicates that serotonin is released in the brain after one or more orgasms during sexual activity (Lorrain et al., 1997, 1999; Pfaus, 2009), which induces sexual satiety and refractoriness (Pfaus, 2009). It is possible that the increase in endogenous serotonin levels that occurs during PVI may mediate the beneficial effects on memory performance through the promotion of neurogenesis. This is especially likely, as studies of rodents that are given selective serotonin reuptake inhibitors have shown that this neurotransmitter promotes neurogenesis (Malberg et al., 2000). Similarly, oxytocin increases during sexual arousal in humans and shows a marked increase during the orgasmic phase (Carmichael et al., 1987). Carmichael, Warburton, Dixen, and Davidson (1994) also reported a correlation between oxytocin levels and the intensity of orgasm in males and females. In women, genital and breast stimulation are particularly potent in inducing its release (Wakerley, Clarke, \& Summerlee, 2006). This chemical has been found to promote neurogenesis even under conditions of stress in rats (Leuner et al., 2012). It is possible, therefore, that increases in oxytocin levels experienced during sex may contribute to promoting neurogenesis in the adult dentate gyrus as well, which could then mediate the beneficial effect on memory performance demonstrated in the current study. 
Additionally, the increase in oxytocin during the orgasmic phase, followed by the post-orgasmic increase in serotonin levels, suggests that orgasm frequency may be an important mediating factor in the posited relationship between PVI frequency, neurogenesis, and memory function. It follows that behavior that maximizes oxytocin and serotonin release, namely PVI that progresses to orgasm, might result in maximal benefit for memory performance. As the current study found a significant relationship between orgasm frequency during PVI and memory performance for those women who do not take oral contraceptives, it is possible that orgasm frequency may mediate the relationship between PVI frequency and memory function through the promotion of serotonin and/or oxytocin release.

As a final point, it is important to consider from an evolutionary standpoint why the relationship between PVI and memory function might exist. Though highly speculative, it can be theorized that those ancestors who engaged in a relatively higher frequency of PVI benefitted from increased neurogenesis, neurotransmitter release, and those neurobiological benefits associated with exercise [such as increased BDNF release (Cotman \& Berchtold, 2002)], since PVI is a form of physical activity. These benefits may have produced bigger, healthier brains and consequently enhanced memory function. Improved memory might have a number of benefits, including a superior ability to remember the location of resources and to engage in more complex procedures for daily life. Thus, it may have eventually contributed to an increased chance of survival. Alternatively, it could be that better memory function evolved as a simple by-product of engaging in PVI. It is plausible that frequent PVI increased the occurrence of the aforementioned neurobiological changes associated with the activity and happened to improve memory function. This enhanced memory function may nothave directly increased fitness, but because it also did not decrease reproductive fitness, the ability was not selected against and selected out of subsequent generations. Both mechanisms potentially explain the existence of the association found in our sample.

Taken together, our results suggest that engaging in sexual intercourse can have beneficial effects for hippocampal-dependent memory, potentially through a mechanism involving neurogenesis in the dentate gyrus. Future studies should investigate the possible neural and hormonal factors that mediate the relationship between PVI and memory performance in order to identify the mechanisms behind this observation.

Acknowledgments We would like to thank Anjali Cera and Kira Riehm for their help in testing participants throughout the study.

Funding This study was funded by a Natural Sciences and Engineering Research Council Discovery Grant (249996) to Jens C. Pruessner. Dorothee Schoemaker was supported by a FRQS doctoral stipend.

\section{Compliance with Ethical Standards}

Conflict of interest The authors declare that they have no conflict of interest.
Ethical Approval All procedures performed in studies involving human participants were in accordance with the ethical standards of the institutional and/or national research committee and with the 1964 Helsinki Declaration and its later amendments or comparable ethical standards.

Informed Consent Informed consent was obtained from all individual participants included in the study.

\section{Appendix}

\section{Select Experience of Intimacy Questionnaire Items}

\section{Sexual Intimacy Items}

Do you engage in sexual intercourse? Yes No

If yes, how often do you engage in sexual intercourse?

1. more than once per day

2. once per day

3. once per week to six times per week

4. once every two-three weeks

5. once per month

6. less than once per month

If yes, does your partner ejaculate inside your vagina, without a condom? Yes_, No

How often does your partner ejaculate inside of you, without a condom, when you engage in sexual intercourse (approximately)?

1. $100 \%$ of the time

2. $75 \%$ of the time

3. $50 \%$ of the time

4. $25 \%$ of the time

5. $\mathrm{n} / \mathrm{a}$

If you engage in sexual intercourse, how often do you achieve orgasm (approximately)?

1. $100 \%$ of the time

2. $75 \%$ of the time

3. $50 \%$ of the time

4. $25 \%$ of the time

5. less than $25 \%$ of the time

6. I do not orgasm during sexual intercourse

How often do you engage in sexual activity other than sexual intercourse? (Including, but not limited to, such activity as oral sex, genito-genital rubbing, genital petting, and mutual masturbation, but excluding kissing.)

1. more than once per day

2. once per day

3. once per week to six times per week

4. once every two-three weeks

5. once per month 
6. less than once per month

7. I do not engage in sexual activity of any kind

Do you masturbate (Yes , No ), and if so, how many times per week?

If you masturbate, how often do you achieve orgasm (approximately)?

1. $100 \%$ of the time

2. $75 \%$ of the time

3. $50 \%$ of the time

4. $25 \%$ of the time

5. I do not orgasm from masturbation

\section{Rubin's Measurement of Romantic Love Scale}

If you are in a monogamous relationship or are seeing a partner on a regular basis (i.e., through casually dating or through a semimonogamous relationship), please indicate the degree of liking and loving you feel toward this partner (referred to below as " $\mathrm{X}$ ") by answering the following questions:

Using the 9-point scale below, please indicate the extent to which you agree or disagree by writing the appropriate number in the space corresponding to each item.

\begin{tabular}{lllllllll}
\hline 1 & 2 & 3 & 4 & 5 & 6 & 7 & 8 & 9 \\
$\begin{array}{l}\text { Not at all true; } \\
\text { disagree } \\
\text { completely }\end{array}$ & & & & & & & & \\
\hline
\end{tabular}

\section{Experience of Loving}

1. ___ If X were feeling badly, my first duty would be to cheer him up.

2. I__ I feel that I can confide in X about virtually everything.

3. __ I find it easy to ignore X's faults.

4. I I would do almost anything for X.

5. _ I I feel very possessive toward X.

6. If_ If I could never be with X, I would feel miserable.

7. ___ If I were lonely, my first thought would be to seek X out.

8. __ One of my primary concerns is X's welfare.

9. I I would forgive $\mathrm{X}$ for practically anything.

10. __ I feel responsible for X's well-being.

11. When I am with X, I spend a good deal of time just looking at him.

12. _ I I would greatly enjoy being confided in by X.

13. It It would be hard for me to get along without $\mathrm{X}$.

\section{References}

Ågmo, A., \& Berenfeld, R. (1990). Reinforcing properties of ejaculation in the male rat: Role of opioids and dopamine. Behavioral Neuroscience, $104,177-182$.

Báez-Mendoza, R., \& Schultz, W. (2013). The role of the striatum in social behavior. Frontiers in Neuroscience. doi:10.3389/fnins.2013.00233.

Becker, J. B., Rudick, C. N., \& Jenkins, W. J. (2001). The role of dopamine in the nucleus accumbens and striatum during sexual behavior in the female rat. Journal of Neuroscience, 21, 3236-3241.

Bedard, A., Gravel, C., \& Parent, A. (2006). Chemical characterization of newly generated neurons in the striatum of adult primates. Experimental Brain Research, 170, 501-512.

Berchtold, N. C., Castello, N., \& Cotman, C. W. (2010). Exercise and timedependent benefits to learning and memory. Neuroscience, 167, 588597.

Blanchflower, D. G., \& Oswald, A. J. (2004). Money, sex and happiness: An empirical study. Scandinavian Journal of Economics, 106, 393-415.

Broadbent, N. J., Gaskin, S., Squire, L. R., \& Clark, R. E. (2010). Object recognition memory and the rodent hippocampus. Learning \& Memory, $17,5-11$.

Brody, S. (2006). Blood pressure reactivity to stress is better for people who recently had penile-vaginal intercourse than for people who had other or no sexual activity. Biological Psychology, 71, 214-222.

Brody, S. (2010). The relative health benefits of different sexual activities. Journal of Sexual Medicine, 7, 1336-1361.

Brody, S., \& Costa, R. M. (2009). Satisfaction (sexual, life, relationship, and mental health) is associated directly with penile-vaginal intercourse, but inversely with other sexual behavior frequencies. Journal of Sexual Medicine, 6, 1947-1954.

Brody, S., \& Krüger, T. H. (2006). The post-orgasmic prolactin increase following intercourse is greater than following masturbation and suggests greater satiety. Biological Psychology, 71, 312-315.

Bruel-Jungerman, E., Laroche, S., \& Rampon, C. (2005). New neurons in the dentate gyrus are involved in the expression of enhanced long-term memory following environmental enrichment. European Journal of Neuroscience, 21, 513-521.

Burleson, M. H., Trevathan, W. R., \& Todd, M. (2007). In the mood for love or vice versa? Exploring the relations among sexual activity, physical affection, affect, and stress in the daily lives of mid-aged women. Archives of Sexual Behavior, 36, 357-368.

Burt, D. B., Zembar, M. J., \& Niederehe, G. (1995). Depression and memory impairment: A meta-analysis of the association, its pattern, and specificity. Psychological Bulletin, 117, 285-305.

Carmichael, M. S., Humbert, R., Dixen, J., Palmisano, G., Greenleaf, W., \& Davidson, J. M. (1987). Plasma oxytocin increases in the human sexual response. Journal of Clinical Endocrinology and Metabolism, $64,27-31$.

Carmichael, M. S., Warburton, V. L., Dixen, J., \& Davidson, J. M. (1994). Relationships among cardiovascular, muscular, and oxytocin responses during human sexual activity. Archives of Sexual Behavior, 23, 59-79.

Carter, C. S. (1992). Oxytocin and sexual behavior. Neuroscience and Biobehavioral Reviews, 16, 131-144.

Clelland, C., Choi, M., Romberg, C., Clemenson, G., Fragniere, A., Tyers, P., ... Gage, F. (2009). A functional role for adult hippocampal neurogenesis in spatial pattern separation. Science, 325, 210-213.

Costa, R. M., \& Brody, S. (2007). Women's relationship quality is associated with specifically penile-vaginal intercourse orgasm and frequency. Journal of Sex and Marital Therapy, 33, 319-327.

Cotman, C. W., \& Berchtold, N. C. (2002). Exercise: A behavioral intervention to enhance brain health and plasticity. Trends in Neurosciences, $25,295-301$ 
Damsma, G., Pfaus, J. G., Wenkstern, D., Phillips, A. G., \& Fibiger, H. C. (1992). Sexual behavior increases dopamine transmission in the nucleus accumbens and striatum of male rats: Comparison with novelty and locomotion. Behavioral Neuroscience, 106, 181-191.

Dayer, A. G., Cleaver, K. M., Abouantoun, T., \& Cameron, H. A. (2005). New GABAergic interneurons in the adult neocortex and striatum are generated from different precursors. Journal of Cell Biology, 168, 415427.

del Rio, J. A., \& Soriano, E. (1989). Immunocytochemical detection of 5'bromodeoxyuridine incorporation in the central nervous system of the mouse. Developmental Brain Research, 49, 311-317.

Diana, R. A., Yonelinas, A. P., \& Ranganath, C. (2007). Imaging recollection and familiarity in the medial temporal lobe: A three-component model. Trends in Cognitive Sciences, 11, 379-386.

Erickson, K. I., Prakash, R. S., Voss, M. W., Chaddock, L., Hu, L., Morris, K. S., ... Kramer, A. F. (2009). Aerobic fitness is associated with hippocampal volume in elderly humans. Hippocampus, 19, 1030-1039.

Eriksson, P. S., Perfilieva, E., Björk-Eriksson, T., Alborn, A.-M., Nordborg, C., Peterson, D. A., \& Gage, F. H. (1998). Neurogenesis in the adult human hippocampus. Nature Medicine, 4, 1313-1317.

Ernst, A., Alkass, K., Bernard, S., Salehpour, M., Perl, S., Tisdale, J., ... Frisén, J. (2014). Neurogenesis in the striatum of the adult human brain. Cell, 156, 1072-1083.

Fernández, G., Weyerts, H., Schrader-Bölsche, M., Tendolkar, I., Smid, H. G., Tempelmann, C., ... Mangun, G. R. (1998). Successful verbal encoding into episodic memory engages the posterior hippocampus: A parametrically analyzed functional magnetic resonance imaging study. Journal of Neuroscience, 18, 1841-1847.

Glasper, E. R., \& Gould, E. (2013). Sexual experience restores age-related decline in adult neurogenesis and hippocampal function. Hippocampus, 23, 303-312.

Gordon, G., Burch, R. L., \& Platek, S. M. (2002). Does semen have antidepressant properties? Archives of Sexual Behavior, 31, 289-293.

Goshen, I., Kreisel, T., Ben-Menachem-Zidon, O., Licht, T., Weidenfeld, J., Ben-Hur, T., \& Yirmiya, R. (2008). Brain interleukin-1 mediates chronic stress-induced depression in mice via adrenocortical activation and hippocampal neurogenesis suppression. Molecular Psychiatry, 13, 717-728.

Gould, E., Beylin, A., Tanapat, P., Reeves, A., \& Shors, T. J. (1999). Learning enhances adult neurogenesis in the hippocampal formation. Nature Neuroscience, 2, 260-265.

Greenwood, B. N., Foley, T. E., Le, T. V., Strong, P. V., Loughridge, A. B., Day, H. E., \& Fleshner, M. (2011). Long-term voluntary wheel running is rewarding and produces plasticity in the mesolimbic reward pathway. Behavioural Brain Research, 217, 354-362.

Hawken, P. A., Jorre, T. J. S., Rodger, J., Esmaili, T., Blache, D., \& Martin, G. B. (2009). Rapid induction of cell proliferation in the adult female ungulate brain (Ovis aries) associated with activation of the reproductive axis by exposure to unfamiliar males. Biology of Reproduction, 80 , 1146-1151.

Haxby, J. V., Ungerleider, L. G., Horwitz, B., Maisog, J. M., Rapoport, S. I., \& Grady, C. L. (1996). Face encoding and recognition in the human brain. Proceedings of the National Academy of Sciences, 93, 922-927.

Heckers, S., Weiss, A. P., Alpert, N. M., \& Schacter, D. L. (2002). Hippocampal and brain stem activation during word retrieval after repeated and semantic encoding. Cerebral Cortex, 12, 900-907.

Heiman, J. R., Long, J. S., Smith, S. N., Fisher, W. A., Sand, M. S., \& Rosen, R. C. (2011). Sexual satisfaction and relationship happiness in midlife and older couples in five countries. Archives of Sexual Behavior, 40, 741-753.

Hurlbert, D. F., \& Whittaker, K.E. (1991). The role of masturbation in marital and sexual satisfaction: A comparative study of female masturbators and nonmasturbators. Journal of Sex Education and Therapy, 17, 272-282.

Jacobs, B., van Praag, H., \& Gage, F. (2000). Adult brain neurogenesis and psychiatry: A novel theory of depression. Molecular Psychiatry, 5, 262-269.
Jessberger, S., Clark, R. E., Broadbent, N. J., Clemenson, G. D., Consiglio, A., Lie, D. C., ... Gage, F. H. (2009). Dentate gyrus-specific knockdown of adult neurogenesis impairs spatial and object recognition memory in adult rats. Learning \& Memory, 16, 147-154.

Kashdan, T. B., Adams, L. M., Farmer, A. S., Ferssizidis, P., McKnight, P.E., \& Nezlek, J. B. (2014). Sexual healing: Daily diary investigation of the benefits of intimate and pleasurable sexual activity in socially anxious adults. Archives of Sexual Behavior, 43, 1417-1429.

Kempermann, G., Kuhn, H. G., \& Gage, F. H. (1997). Genetic influence on neurogenesis in the dentate gyrus of adult mice. Proceedings of the National Academy of Sciences, 94, 10409-10414.

Kennedy, K. M., Hope, K., \& Raz, N. (2009). Life span adult faces: Norms for age, familiarity, memorability, mood, and picture quality. Experimental Aging Research, 35, 268-275.

Kim, J.-I., Lee, J. W., Lee, Y. A., Lee, D.-H., Han, N. S., Choi, Y.-K., . .. Han, J. S. (2013). Sexual activity counteracts the suppressive effects of chronic stress on adult hippocampal neurogenesis and recognition memory. Brain Research, 1538, 26-40.

Kuhn, H. G., Dickinson-Anson, H., \& Gage, F.H. (1996). Neurogenesis in the dentate gyrus of the adult rat: Age-related decrease of neuronal progenitor proliferation. Journal of Neuroscience, 16, 2027-2033.

Laumann, E. O., Paik, A., Glasser, D. B., Kang, J.-H., Wang, T., Levinson, B., ... Gingell, C. (2006). A cross-national study of subjective sexual wellbeing among older women and men: Findings from the Global Study of Sexual Attitudes and Behaviors. Archives of Sexual Behavior, 35, 143159

Leuner, B., Caponiti, J. M., \& Gould, E. (2012). Oxytocin stimulates adult neurogenesis even under conditions of stress and elevated glucocorticoids. Hippocampus, 22, 861-868.

Leuner, B., Glasper, E. R., \& Gould, E. (2010). Sexual experience promotes adult neurogenesis in the hippocampus despite an initial elevation in stress hormones. PLOS ONE, 5(7), e11597.

Levin, R. J. (2007). Sexual activity, health and well-being-The beneficial roles of coitus and masturbation. Sexual and Relationship Therapy, 22, $135-148$.

Lorrain, D. S., Matuszewich, L., Friedman, R. D., \& Hull, E. M. (1997). Extracellular serotonin in the lateral hypothalamic area is increased during the postejaculatory interval and impairs copulation in male rats. Journal of Neuroscience, 17, 9361-9366.

Lorrain, D. S., Riolo, J. V., Matuszewich, L., \& Hull, E. M. (1999). Lateral hypothalamic serotonin inhibits nucleus accumbens dopamine: Implications for sexual satiety. Journal of Neuroscience, 19, 7648-7652.

Mak, G. K., Enwere, E. K., Gregg, C., Pakarainen, T., Poutanen, M., Huhtaniemi, I., \& Weiss, S. (2007). Male pheromone-stimulated neurogenesis in the adult female brain: Possible role in mating behavior. Nature Neuroscience, 10, 1003-1011.

Malberg, J. E., Eisch, A. J., Nestler, E. J., \& Duman, R. S. (2000). Chronic antidepressant treatment increases neurogenesis in adult rat hippocampus. Journal of Neuroscience, 20, 9104-9110.

Manganas, L. N., Zhang, X., Li, Y., Hazel, R. D., Smith, S. D., Wagshul, M. E., ... Enikolopov, G. (2007). Magnetic resonance spectroscopy identifies neural progenitor cells in the live human brain. Science, 318, 980985 .

Marlatt, M. W., Potter, M. C., Lucassen, P. J., \& van Praag, H. (2012). Running throughout middle-age improves memory function, hippocampal neurogenesis, and BDNF levels in female C57BL/6 J mice. Developmental Neurobiology, 72, 943-952.

Meijer, J. H., \& Robbers, Y. (2014). Wheel running in the wild. Proceedings of the Royal Society of London B: Biological Sciences, 281, 20140210.

Mermelstein, P. G., \& Becker, J. B. (1995). Increased extracellular dopamine in the nucleus accumbens and striatum of the female rat during paced copulatory behavior. Behavioral Neuroscience, 109, 354-365.

Ming, G.-L., \& Song, H. (2011). Adult neurogenesis in the mammalian brain: Significant answers and significant questions. Neuron, 70, 687-702.

Mirescu, C., \& Gould, E. (2006). Stress and adult neurogenesis. Hippocampus, 16, 233-238. 
Moncho-Bogani, J., Martinez-Garcia, F., Novejarque, A., \& Lanuza, E. (2005). Attraction to sexual pheromones and associated odorants in female mice involves activation of the reward system and basolateral amygdala. European Journal of Neuroscience, 21, 2186-2198.

Moraska, A., Deak, T., Spencer, R. L., Roth, D., \& Fleshner, M. (2000). Treadmill running produces both positive and negative physiological adaptations in Sprague-Dawley rats. American Journal of Physiology, 279, R1321-R1329.

Nakashiba, T., Cushman, J. D., Pelkey, K. A., Renaudineau, S., Buhl, D. L., McHugh, T. J., ... McBain, C. J. (2012). Young dentate granule cells mediate pattern separation, whereas old granule cells facilitate pattern completion. Cell, 149, 188-201.

Nestor, A., Plaut, D. C., \& Behrmann, M. (2011). Unraveling the distributed neural code of facial identity through spatiotemporal pattern analysis. Proceedings of the National Academy of Sciences, 108, 9998-10003.

Ormerod, B., \& Galea, L. (2001). Reproductive status influences cell proliferation and cell survival in the dentate gyrus of adult female meadow voles: A possible regulatory role for estradiol. Neuroscience, 102, 369379.

Paivio, A., Yuille, J.C., \& Madigan, S. A. (1968). Concreteness, imagery, and meaningfulness values for 925 nouns. Journal of Experimental Psychology, 76, 1-25.

Pereira, A. C., Huddleston, D. E., Brickman, A. M., Sosunov, A. A., Hen, R., McKhann, G. M., ... Small, S. A. (2007). An in vivo correlate of exercise-induced neurogenesis in the adult dentate gyrus. Proceedings of the National Academy of Sciences, 104, 5638-5643.

Pfaus, J. G. (2009). Pathways of sexual desire. Journal of Sexual Medicine, 6, $1506-1533$.

Pfaus, J. G., Damsma, G., Wenkstern, D., \& Fibiger, H. (1995). Sexual activity increases dopamine transmission in the nucleus accumbens and striatum of female rats. Brain Research, 693, 21-30.

Richardson, M., Abraham, C., \& Bond, R. (2012). Psychological correlates of university students" academic performance: A systematic review and meta-analysis. Psychological Bulletin, 138, 353-387.

Rossion, B., Caldara, R., Seghier, M., Schuller, A. M., Lazeyras, F., \& Mayer, E. (2003). A network of occipito-temporal face-sensitive areas besides the right middle fusiform gyrus is necessary for normal face processing. Brain, 126, 2381-2395.

Rossion, B., Hanseeuw, B., \& Dricot, L. (2012). Defining face perception areas in the human brain: A large-scale factorial fMRI face localizer analysis. Brain and Cognition, 79, 138-157.
Rowland, D. L. (2006). Neurobiology of sexual response in men and women. CNS Spectrums, 11(S9), 6-12.

Rubin, Z. (1970). Measurement of romantic love. Journal of Personality and Social Psychology, 16, 265-273.

Sahay, A., Scobie, K. N., Hill, A. S., O'’arroll, C. M., Kheirbek, M. A., Burghardt, N. S., ... Hen, R. (2011). Increasing adult hippocampal neurogenesis is sufficient to improve pattern separation. Nature, 472, 466-470.

Shulman, J. L., \& Horne, S. G. (2003). The use of self-pleasure: Masturbation and body image among African American and European American women. Psychology of Women Quarterly, 27, 262-269.

Spalding, K. L., Bergmann, O., Alkass, K., Bernard, S., Salehpour, M., Huttner, H. B., ... Buchholz, B. A. (2013). Dynamics of hippocampal neurogenesis in adult humans. Cell, 153, 1219-1227.

Spencer, J. P. (2010). The impact of fruit flavonoids on memory and cognition. British Journal of Nutrition, 104(S3), S40-S47.

Stranahan, A. M., Khalil, D., \& Gould, E. (2006). Social isolation delays the positive effects of running on adult neurogenesis. Nature Neuroscience, 9, 526-533.

Takahashi, T.,Zhu, Y.,Hata, T., Shimizu-Okabe, C., Suzuki, K., \& Nakahara, D. (2009). Intracranial self-stimulation enhances neurogenesis in hippocampus of adult mice and rats. Neuroscience, 158, 402-411.

Takamura, N., Nakagawa, S., Masuda, T., Boku, S., Kato, A., Song, N., ... Koyama, T. (2014). The effect of dopamine on adult hippocampal neurogenesis. Progress in Neuro-Psychopharmacology and Biological Psychiatry, 50, 116-124.

van Praag, H., Kempermann, G., \& Gage, F. H. (1999). Running increases cell proliferation and neurogenesis in the adult mouse dentate gyrus. Nature Neuroscience, 2, 266-270.

van Praag, H., Schinder, A.F., Christie, B. R., Toni, N.,Palmer, T. D., \& Gage, F. H. (2002). Functional neurogenesis in the adult hippocampus. Nature, $415,1030-1034$.

van Praag, H., Shubert, T., Zhao, C., \& Gage, F. H. (2005). Exercise enhances learning and hippocampal neurogenesis in aged mice. Journal of Neuroscience, $25,8680-8685$.

Wadsworth, T. (2014). Sex and the pursuit of happiness: How other people's sex lives are related to our sense of well-being. Social Indicators Research, $116,115-135$.

Wakerley, J., Clarke, G., \& Summerlee, A. (2006). Milk ejection and its control. In E. Knobil \& J. Neill (Eds.), The physiology of reproduction (3rd ed., Vol. 2, pp. 3129-3190). New York: Raven Press. 